\title{
Prevalence of Mycobacterium tuberculosis in Children Suffering from Lower Respiratory Tract Infection Attending a Tertiary Care Hospital, Hyderabad
}

\author{
Vipparti Haritha $^{1^{*}}$ and V.V. Shailaja ${ }^{2}$ \\ ${ }^{1}$ Department of Microbiology, Malla Reddy Institute of Medical Sciences, \\ Hyderabad, Telangana state, India \\ ${ }^{2}$ Department of Microbiology, Gandhi Medical College, Hyderabad, Telangana state, India \\ *Corresponding author
}

\section{A B S T R A C T}

Though the declaration by the WHO of a 'global TB emergency' was in 1993, the emphasis has been more on adult disease. Pediatric TB was neglected, mainly due to

\section{Keywords}

Gastric aspirates, Mycobacterium, Drug susceptibility

Article Info

Accepted:

23 June 2017

Available Online:

10 July 2017 greater challenges in diagnosis and the lower priority traditionally afforded to children by TB control programmes. As a result both research and surveillance data in the field of childhood TB have been greatly limited. Hence the present study was undertaken from $6 / 1 / 10$ - 8/2/11 to know the isolation rate of Mycobacterium tuberculosis and Drug susceptibility patterns in children suffering from LRTI. 153 Gastric aspirates were collected and processed by conventional methods and anti-tubercular drug sensitivity testing was done by Proportion method. 30 isolates of Mycobacterium were obtained of which 7 isolates were Atypical Mycobacteria. All isolates of Mycobacterium tuberculosis were sensitive to Isoniazid, Rifampicin and Ethambutol. 13.6\% of the strains were resistant to Streptomycin. TB in children presents particularly difficult challenges, but research priorities and advances in pediatric TB research may also provide wider insights and opportunities for TB control. Better diagnostics would probably represent the next most important step which will not only improve individual case management, but also provide a rapid detection of drug resistance.

\section{Introduction}

Mycobacterium tuberculosis is an important agent causing LRTI. Pediatric tuberculosis has traditionally received a lower priority than adult TB in National TB programmes because it is considered non-infectious, is difficult to diagnose, cases have been thought to be few and it was wrongly assumed that effective control of adult TB and use of BCG by itself could prevent childhood TB. In India, there are about $\sim 400$ million children who constitute about $34 \%$ of the total population. The extent of childhood $\mathrm{TB}$ in India is estimated to be $10.2 \%$ of the total adult incidence. Globally, about 1 million cases of paediatric $\mathrm{TB}$ are estimated to occur every year accounting for $10-15 \%$ of all TB. Though MDR-TB and XDR-TB is documented among paediatric age group, there are no estimates of overall burden, chiefly because of diagnostic difficulties and exclusion of children in most of the drug resistance surveys (1). The proportion of pediatric TB cases registered under RNTCP has shown an increasing trend, from $5.6 \%$ 
(59846 cases) in 2005 to $7 \%$ (84064 cases) in 2011 (1).

In brief, $\mathrm{TB}$ diagnosis is based on clinical features, smear examination of sputum where this is available, positive family history, tuberculin skin testing, chest radiography and histo pathological examination.

The present study was undertaken to note the prevalence of Mycobacterium tuberculosis causing LRTI in children.

\section{Materials and Methods}

This study was undertaken to determine the prevalence of Mycobacterium tuberculosis and their anti-tubercular drug susceptibility pattern in children.

\section{Place of study}

In a tertiary care hospital in Hyderabad, India

\section{Duration}

The present study was undertaken for a period of one year i.e. from $6 / 1 / 10-8 / 2 / 11$

\section{Study group}

Comprises of children clinically diagnosed as cases of Lower Respiratory Tract Infection (LRTI) belonging to age group $0-4$ years.

\section{Specimen}

Gastric aspirate from children (two samples were collected on two consecutive days).

\section{Processing}

Gastric aspirates were decontaminated by Modified Petroff's method before inoculating on LJ media and for smear preparation (Figure 1).
Sample was then processed as follows (Figure 2).

L-J slants were incubated for 8 weeks. Reading was first taken after $72 \mathrm{hrs}$ and weekly thereafter for 8 weeks.

Isolates which showed growth on LJ slant within 1 week after inoculation were cultured on LJ slant with PNB (p-nitro benzoate). If growth was present on LJ slant with PNB they were regarded as Atypical Mycobacteria and were not processed further.

Mycobacterium tuberculosis isolates without any growth on LJ slant with PNB were further considered for drug susceptibility testing. Drug susceptibility testing of Mycobacterial isolates obtained was determined by the Proportion Method.

Control strains used in the study: $\mathrm{M}$. tuberculosis H37Rv.

\section{Anti-tubercular drug sensitivity testing}

Drug susceptibility testing of Mycobacterial isolates obtained was determined by the Proportion Method in State TB centre Erragadda, Hyderabad, Telangana state, India.

\section{Criteria of resistance}

Any strain with $1 \%$ (the critical proportion) of bacilli resistant to any of the four drugs Rifampicin, Isoniazid, Ethambultol, and streptomycin - is classified as resistant to that drug (2).

\section{Results and Discussion}

The total number of children suspected of Tuberculosis included in the study during the one year period was 153 (Figure 3). Smear was positive for Acid Fast Bacilli by Zeihl Neelsen's staining in 65 cases $(42.4 \%)$, from 
which Mycobacteria was isolated on LJ slant in 30 cases (19.6\%) (Figures 4 and 5).

When processed on LJ slant with PNB, 22 isolates of Mycobacterium tuberculosis and 8 Atypical Mycobacteria were obtained. Atypical Mycobacteria were not subjected for sensitivity testing. 22 isolates of Mycobacterium tuberculosis were further processed for anti-tubercular drug susceptibility testing (Figure 6 and Table 1).

TB in children is often missed or overlooked due to non-specific symptoms and difficulties in diagnosis. This has made it difficult to assess the actual magnitude of the childhood TB epidemic, which may be higher than currently estimated.

In the present study among all the gastric aspirate specimens obtained, smear for Acid Fast Bacilli were positive in $42.4 \%$ and culture for Mycobacteria on LJ slants was positive in 30(19.6\%) isolates (Tables 2).

\section{Relationship with age}

The high incidence of tuberculosis in early childhood appears to be related to the low resistance of the host and serious manifestation of the disease at this age. O. N. Bhakoo and Gupta (1969) observed that $71 \%$ of smear positive cases were children belonging to 0-5 yrs age group (9). Similar was the observation by Josephine $M$ Nantongo et al., (2013) in Uganda where Age group less than 1 year and 1 to 5 years were more likely to be associated with pulmonary TB compared to those children over 5 years of age (7).

In the present study since gastric aspirates were collected from children belonging to age group 0-5 years only, a definitive conclusion regarding the age of the child and positivity rate could not be obtained. However the above studies proved that, $0-5$ yrs is the age group with highest positivity rate.

Smear microscopy and culture on Lowenstein-Jensen solid medium is still the most used amongst all methods employed worldwide due to its simplicity, low cost, speed and minimal requirement of equipment and technical skills. Hence a more sensitive smear microscopy and decontamination method would both be useful for accurate and rapid TB diagnosis.

For effective isolation of Mycobacteria the specimens have to be subjected to various decontamination techniques. In the present study, Modified Petroff's method was used as a decontamination procedure. In a study done by Kiran Tripathi et al., on sputum samples of 225 suspected tuberculosis cases which were processed by petroff's and Modified Petroff's method separately found that $123(54.67 \%)$ were smear positive by direct, 127 (56.44\%) by Petroff's method and $129(57.33 \%)$ by Modified Petroff's method. The number of culture positives by Modified Petroff's method was $55.56 \%$, by Petroff's method were $53.33 \%$ and $45.33 \%$ by Direct method. This shows that modified Petroffs method is a sensitive method for isolating of Mycobacteria. (10)

The main impediment to the accurate diagnosis of active TB is the paucibacillary nature of the disease in children. Younger children also produce smaller amounts of sputum, which is usually swallowed rather than expectorated.

Consequently bacteriological confirmation is the exception rather than the rule with only $10-15 \%$ of sputum samples revealing acid fast bacilli (AFB) and culture remaining negative in around $70 \%$ of cases with probable TB (11). In the present study smear positive -culture negative cases were 24 .

Samples producing a positive AFB smear but 
negative Mycobacterial culture were false positive AFB smear, and pathogen may classified into one of four categories: show a positive AFB smear other than laboratory failure to isolate Mycobacteria, Mycobacteria, and indeterminate results. (12)

Table.1 Anti tubercular drug sensitivity pattern

\begin{tabular}{|l|c|c|}
\hline DRUG & SENSITIVE & RESISTANT \\
\hline STREPTOMICIN & $86.4 \%$ & $13.6 \%$ \\
\hline ISONIAZID & $100 \%$ & $0 \%$ \\
\hline RIFAMPICIN & $100 \%$ & $0 \%$ \\
\hline ETHAMBUTOL & $100 \%$ & $0 \%$ \\
\hline
\end{tabular}

Table.2 Comparison of smear and cultures in the present study with various other studies

\begin{tabular}{|l|l|l|}
\hline & Smear positive & Culture positive \\
\hline Present study & $42.4 \%$ & $19.6 \%$ \\
\hline Myrna T. Mendoza et al., (3) & Data not available & $13.09 \%$ \\
\hline Douglas F. Moore (4) & Data not available & $6.9 \%$ \\
\hline Andrea T et al., (5) & Data not available & $11 \%$ \\
\hline .Dilek Saka et al., (6) & $30.9 \%$ & $43.6 \%$ \\
\hline $\begin{array}{l}\text { Josephine M Nantongo et al., } \\
\text { (2013) }\end{array}$ & Data not available & $6.3 \%$ \\
\hline Jithendra Kandati,et al., (8) & Data not available & $44.8 \%$ \\
\hline
\end{tabular}

Table.3 Anti Mycobacterial drug resistance seen in various studies

\begin{tabular}{|l|l|l|l|l|}
\hline & $\begin{array}{l}\text { Culture } \\
\text { positive }\end{array}$ & Sensitive & Mono resistance & $\begin{array}{l}\text { Multidrug } \\
\text { resistance }\end{array}$ \\
\hline Present study & $19.6 \%$ & I, R,E -100\% & S -13.6\% & - \\
\hline $\begin{array}{l}\text { ParvanehBaghaei, et al., } \\
(17)\end{array}$ & $48(85.7 \%)$ & I,R,E -87\% & 5 cases & $2.2 \%$ \\
\hline Mark N. Lobato et al., (18) & & & I-9\% & \\
\hline M.Varma et al., (19) & & & I-50\% R-30\% & $22 \%$ \\
\hline Minwuyelet Maru etal (20) & & I, R, E, Z-87\% & & $17 \%$ \\
\hline Ellis Owusu-Dabo (21) & & & S- 23.5\% & \\
\hline
\end{tabular}

I-Isoniazid R- Rifampicin E-Etambutol Z-Pyrazinamide S- Streptomycin 


\section{Figure 1-Modified petroff's method}

Procedure:

To $\mathrm{x} \mathrm{ml}$ of sputum, $2 \mathrm{xml}$ of $4 \% \mathrm{NaOH}$ is added

Tighten cap of container and shake well to digest.

Let it stand for 15 minutes at room temperature with occasional shaking

Centrifuge at $3000 \mathrm{xg}$ for 15 minutes.

supernatant fluid is discarded.

$15 \mathrm{ml}$ sterile saline or distilled water is added to the sediment

Centrifuge at $3000 \mathrm{xg}$ for 15 minutes

$\downarrow$

Decant supematant and inoculate onto culture medium and smear made.

\section{Figure 2 - Processing of gastric aspirates}

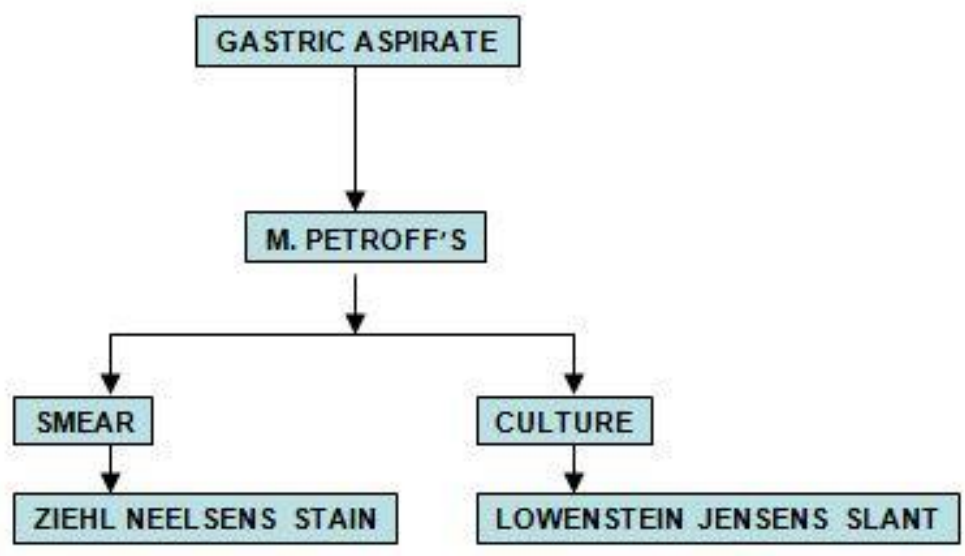


Figure 3-Sex distribution of cases|

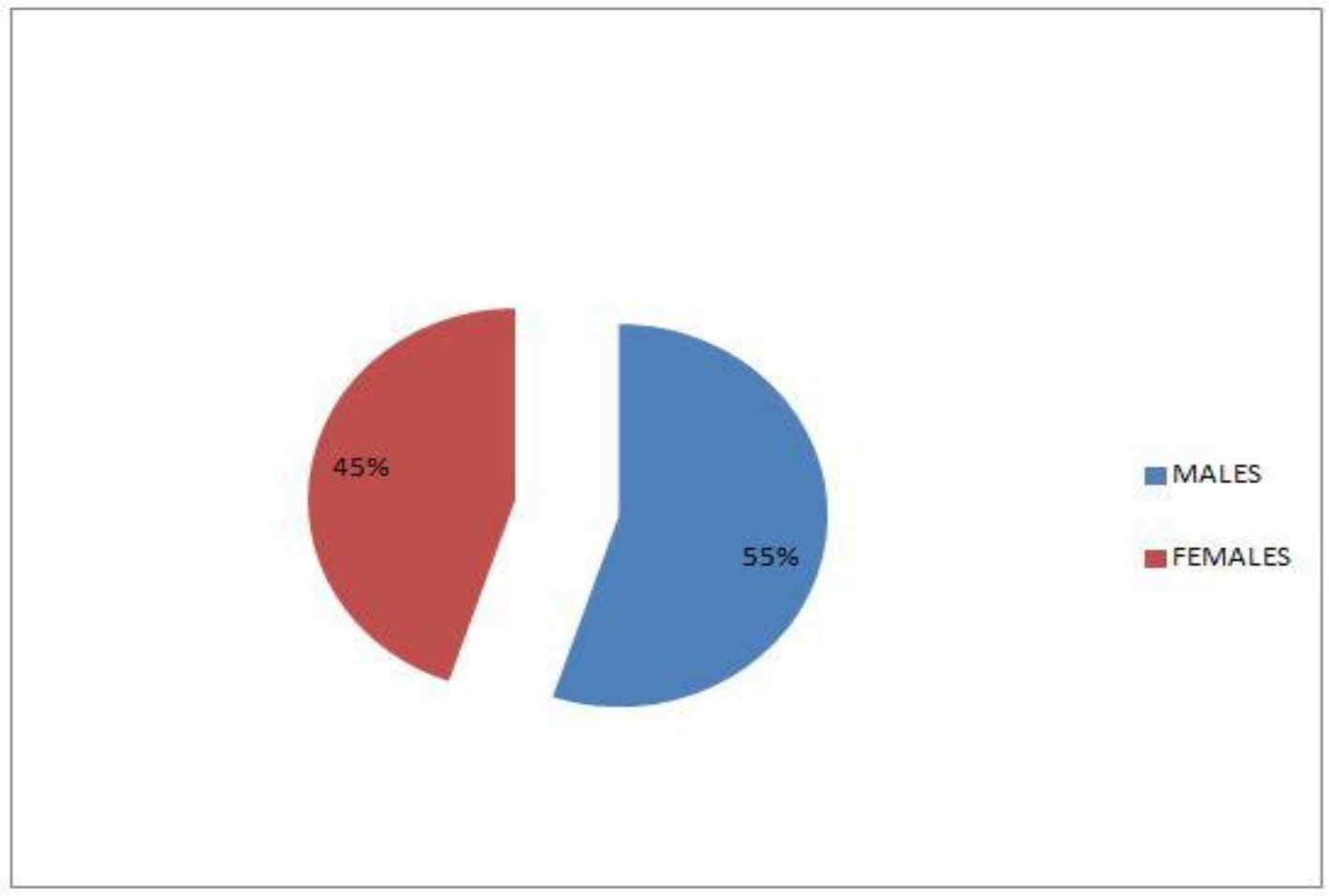

Figure 4- Percentage of Mycobacteria observed in smear and isolated from culture

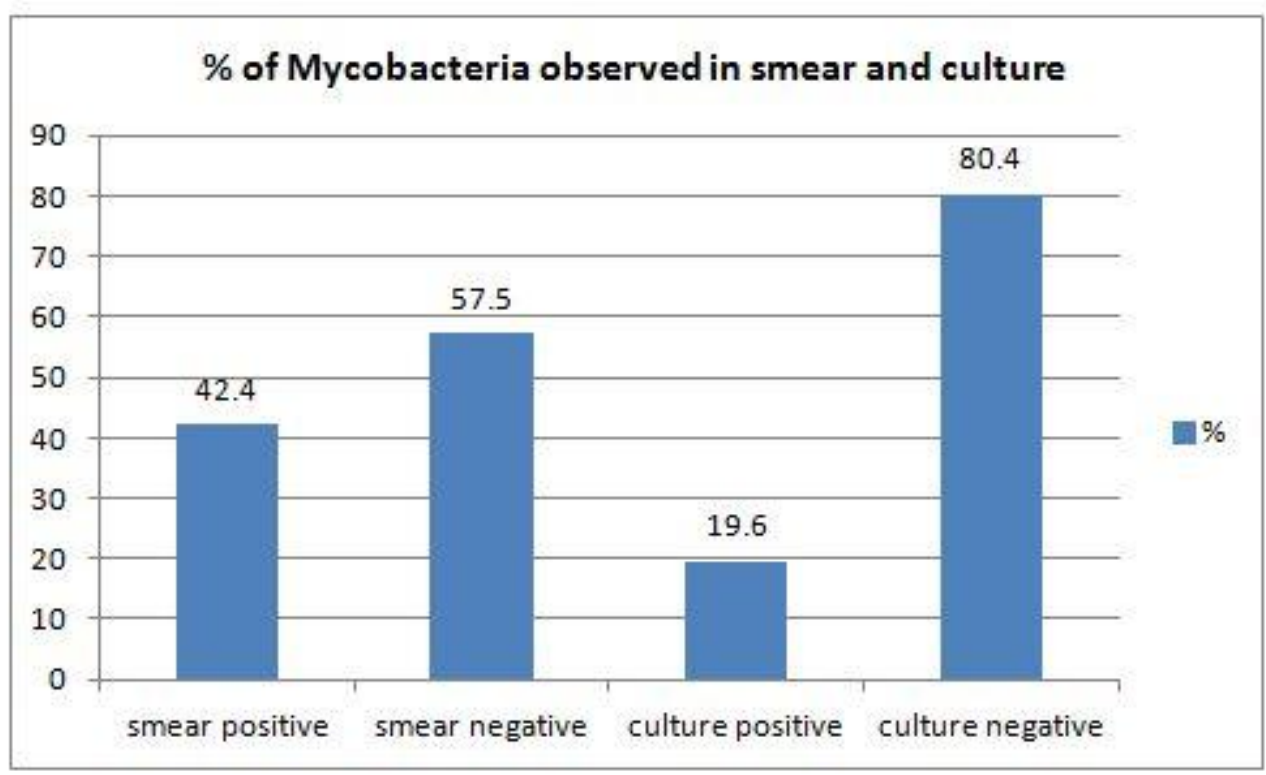


Figure 5-Correlation between smear and culture.|

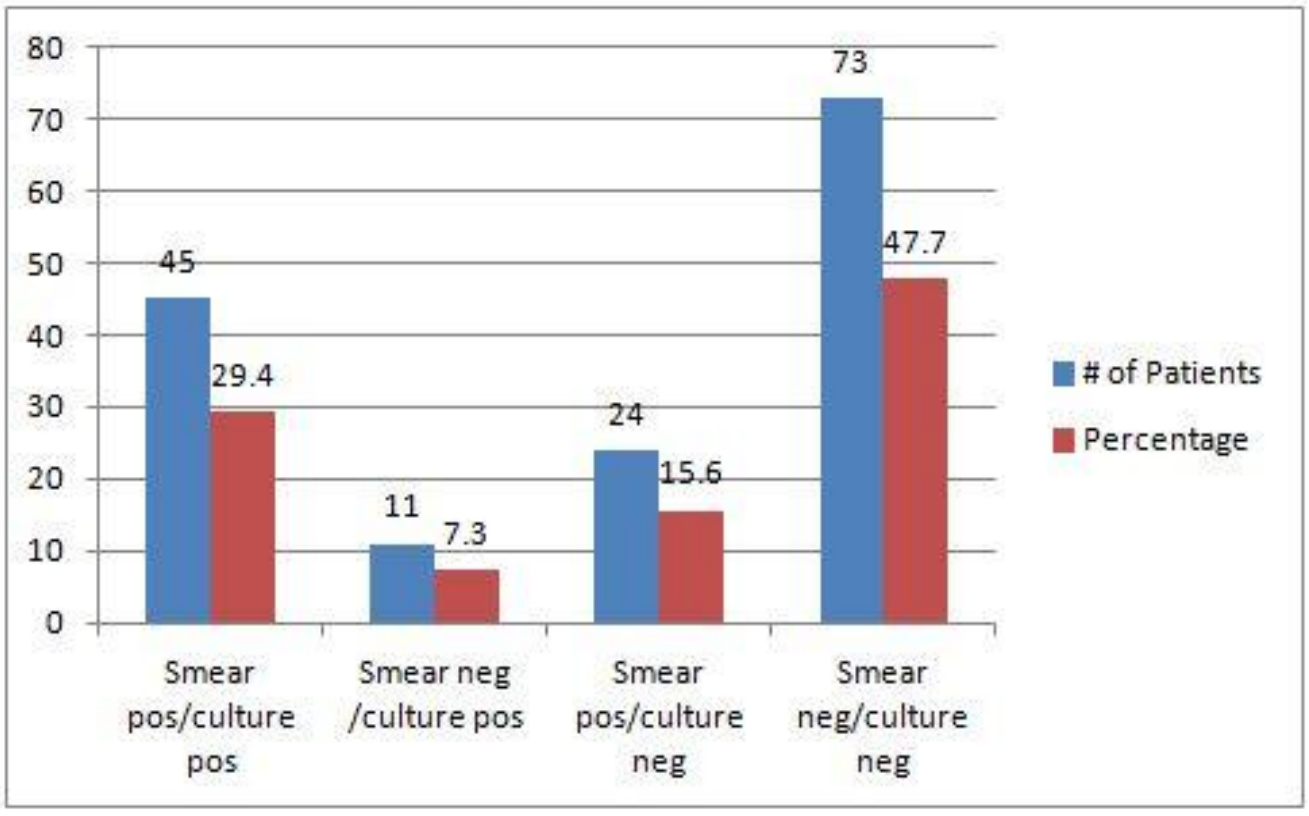

Figure 6-Breakup of Mycobacterium isolates

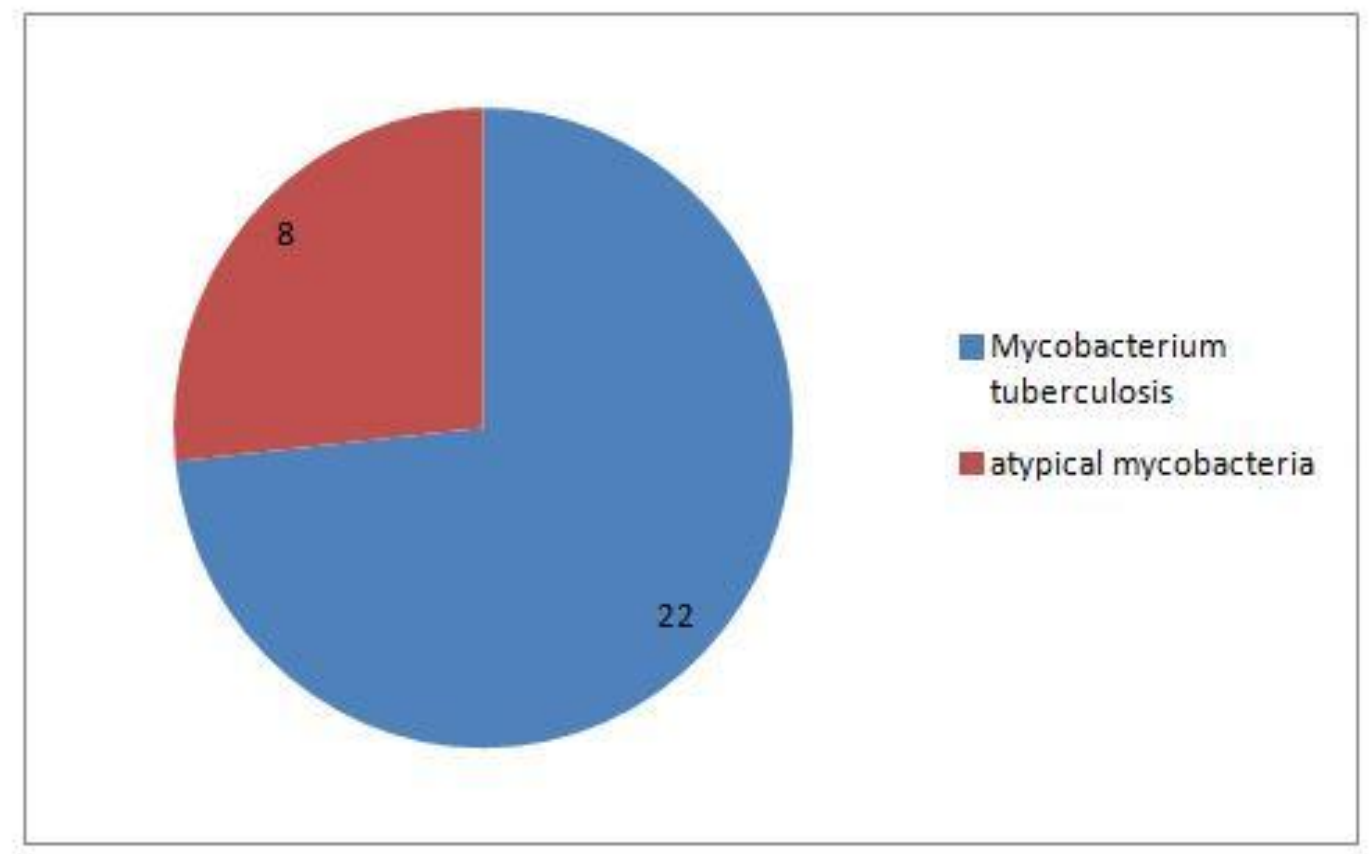


Another reason for the above condition is continued expectoration of dead organisms beyond the period of true culture conversion commonly seen in patients with a heavy pretreatment bacillary load. Smear positive culture negative samples are also seen from 15 to $20 \%$ among samples received during treatment of patients with Rifampicin containing regimens.

According to the WHO definition, Smear negative pulmonary tuberculosis is defined as follows: a. Pulmonary tuberculosis cases with negative sputum smears but growth positive in cultures; b. The patients who meet all of the four criteria stated below: 1. At least two negative sputum smears, 2. Radiological findings consistent with radiologically active pulmonary tuberculosis, 3 . No response to a course of broad-spectrum antibiotics 4. A decision made by a clinician for the treatment with anti-tuberculosis medication.

Smear negative and culture positive cases seen in the present study were 11.8 of these 11 children were started with anti-tubercular therapy because they had significant chest $X$ ray findings consistent with pulmonary tuberculosis.

It is very important to identify such smear negative cases because the relative transmission rate from patients with smearnegative compared with smear-positive PTB is around 22\%. (12). In a study done by Leandro Cruz Campos et al., who studied 198 patients who were positive for culture for Mycobacterium tuberculosis. Of these patients, $69(34.8 \%)$ were smear positive and $129(65.2 \%)$ were smear negative. (13)

$7(31.82 \%)$ isolates which showed growth on LJ slant with PNB were identified as Atypical Mycobacteria and were not processed further. Blyth, Christopher C et al., and Elisa LópezVarela, et al., isolated Atypical Mycobacteria in $35.4 \%$ and $26.3 \%$ of cases respectively (15, 16). 22 isolates of mycobacterium tuberculosis were subjected for drug susceptibility testing.

Laboratory tests for the sensitivity of tubercle bacilli to chemotherapeutic drugs serve three main purposes: first, they can be used as guidance in choice of first course chemotherapy to be given to the patient. Secondly, they may be of value in confirming that drug resistance has emerged when a patient has failed to show a satisfactory bacteriological response to treatment, and may guide the choice of further course of treatment with different drugs. Thirdly, they may be employed to estimate the prevalence of primary and acquired drug resistance in a community.

The value of Anti-Tuberculosis drugs for the treatment of infections caused by Atypical Mycobacteria is limited by the high prevalence of drug resistance. Atypical Mycobacteria are high heterogeneous in their drug sensitivity patterns and in this respect each strain virtually represents a distinct biological unit. No anti-tubercular sensitivity testing was done for the atypical mycobacterium isolates obtained in the present study.

Out of 22 isolates of Mycobacterium tuberculosis 3 isolates showed resistance to streptomycin. Rest of the isolates was sensitive to Streptomicin, Rifampicin, Ethambutol and Isoniazid. The overall prevalence of drug resistance was $13.6 \%$. The following table shows the sensitivity pattern of Mycobacterium tuberculosis isolates obtained in the present study with other studies (Table 3).

Drug resistance pattern is very important factor which has to be monitored particularly in children for three reasons: to determine 
response to therapy; to identify adverse events early; and to promote adherence.

In conclusion, Diagnosis of $\mathrm{TB}$ is very challenging in young children, and Refinement of existing tools is urgently required to improve diagnosis and treatment of TB in children. Higher global priority and funding will be required to reduce the unnecessary and avoidable morbidity and mortality occurring currently.

In addition to reducing the burden of adult TB, attention to childhood nutrition and improvement in the socioeconomic and environmental condition of communities is likely to have a significant impact on TB transmission to children.

\section{References}

1. Ashok Kumar, Devesh Gupta, Sharath Burugina Nagaraja, Varinder Singh, GR Sethi and Jagadish Prasad. Updated National Guidelines for Pediatric Tuberculosis in India, 2012. Indian jurnal of pediatrics 2013: 50: 30-306.

2. Mohammed Rahbar, Masood hajia.Role of gastric lavage for diagnosis of pulmonary tuberculosis. Pakistan journal of medical sciences. Volume 23: 2007.

3. Myrna T. Mendoza, M.D. and Cristino P. Narciso: The reliability of sputum AFB microscopy: 2nd Prize, PSMIDHoechst Annual Research Contest. Microbiology Section, Department of Pathology, Lung Centre of the Philippines.

4. Douglas F. Moore and Janis I. Curry. Detection and Identification of Mycobacterium tuberculosis directly from Sputum Sediments by Ligase Chain Reaction. Journal of Clinical Microbiology. 1998 Apr; 36(4): 10281031.
5. Andrea T. Cruz Paula A. Revell Jeffrey R. Starke. Gastric Aspirate Yield for Children with Suspected Pulmonary Tuberculosis. Journal of Pediatric Infectious Diseases Society. 2013; 2 (2): 171-174. doi: https://doi.org/10.1093/ jpids/pis089.

6. Dilek Saka, Haluk Celalettin Çalışır, Mihriban etal. The diagnostic role of gastric aspiration in cases without sputum and in smear - negative patients with suspected pulmonary Tuberculosis. Journal of tuberculosis and thorax.

7. Josephine $M$ Nantongo, Eric Wobudeya, Ezekiel Mupere, Moses Joloba, Willy Ssengooba, Harriet N Kisembo,et al., High incidence of pulmonary tuberculosis in children admitted with severe pneumonia in Uganda. Bio Med Central Pediatrics201313:16.

8. Jithendra Kandati, Suresh Kumar Boorsu, Muni lakshmi Ponugoti, Vedadruthy Samudrala et al., Bacterial and fungal agents causing lower respiratory tract infections in patients with human immunodeficiency virus infection. http://dx.doi.org/10.18203/ 2320-6012.ijrms20162335.

9. Bhakoo and S. Gupta: tuberculosis in children. Indian journal of pediatrics: 36:65, 1969.

10. Kiran Tripathi1, Purti C. Tripathi, Shashwati Nema, Arun Kumar Shrivastava, Kalpana Dwiwedi5, Ashok Kumar Dhanvijay. Modified Petroff's Method: an Excellent Simplified Decontamination Technique in Comparison with Petroff's Method. International Journal of Recent Trends in Science And Technology, Volume 10, Issue 3, 2014 pp 461-464.

11. Newton $\mathrm{SM}, \mathrm{PhD}$, Brent AJ, MRCP, Anderson S, MRCPCH, Whittaker E, $\mathrm{MRCPCH}, 1$ and Kampmann B, FRCPCH. Paediatric Tuberculosis. 
Lancet Infectious Diseases. 2008 Aug; 8(8): 498-510. doi: $10.1016 /$ S14733099(08)70182-8.

12. Lee JS1, Kim EC, Joo SI, Lee SM, Yoo CG, Kim YW, Han SK, Shim YS, Yim JJ.The incidence and clinical implication of sputum with positive acid-fast bacilli smear but negative in mycobacterial culture in a tertiary referral hospital in South Korea Journal of Korean Medical Sciences.. 2008 Oct; 23(5):767-71. doi: $10.3346 / \mathrm{jkms} .2008$. 23.5.767.

13. Behr MA. Transmission of MTB from patients smear negative for AFB. Lancet 1999;353:444

14. Leandro Cruz Campos, Marcos Vinícius Vieira Rocha, Denise Maria Cunha Willers, Denise Rossato RESEARCH ARTICLE. Characteristics of Patients with Smear-Negative Pulmonary Tuberculosis (TB) in a Region with High TB and HIV Prevalence. PLoS One. 2016; 11(1): e0147933. doi: 10.1371/journal.pone.0147933.

15. Blyth, Christopher C. Best, Emma J. BHB: Nontuberculous Mycobacterial Infection in Children: A Prospective National Study Pediatric Infectious Disease Journal: September 2009 Volume 28 - Issue 9 - pp 801-805.

16. Elisa López-Varela, Alberto L. GarcíaBasteiro, Orvalho J. Augusto, Oscar Fraile,1Helder Bulo et al., High Rates of Non-Tuberculous Mycobacteria Isolation in Mozambican Children with Presumptive Tuberculosis. PMCID: PMC5240942.
17. Parvaneh Baghaei, Payam Tabarsi, Parisa Farnia, Amir Hoseyn Radaei, Mehdi Kazempour et al.,Utility of Gastric Lavage for Diagnosis of Tuberculosis in Patients who are Unable to Expectorate Sputum. Journal of Global Infectious Diseases. 2011 3(4): 339-343.

18. Mark N. Lobato, MD Ann M. Loeffler, MD Karen Furst, MD, MPHi; Barbara Cole, RN, PHN et al., Detection of Mycobacterium tuberculosis in Gastric Aspirates Collected from Children: Hospitalization Is Not Necessary. Pediatrics journal. 1998;102;e40

19. M. Varma, S. Kumar, A. Kumar and M. Bose. Comparison of e test and agar proportion method of testing drug susceptibility of $M$. tuberculosis. Indian Journal of Tuberculosis; 2002,490,217.

20. Minwuyelet Maru, Solomon H. Mariam, Tekle Airgecho, Endalamaw Gadissa, and Abraham Aseffa. Prevalence of Tuberculosis, Drug Susceptibility Testing, and Genotyping of Mycobacterial Isolates from Pulmonary Tuberculosis Patients in Dessie, Ethiopia Volume 2015 (2015), Article ID 215015, 10 pages.doi.org/10.1155/2015/215015.

21. Ellis Owusu-Dabo, Ohene Adjei, Christian G. Meyer, Rolf D. Horstmann, Anthony Enimil, Thomas F. Kruppa, Frank Bonsu et al., Mycobacterium tuberculosis Drug Resistance, Ghana) Emerging Infectious Diseases. $2006 \mathrm{Jul}$; 12(7): 1170-1172.

\section{How to cite this article:}

Vipparti Haritha and Shailaja, V.V. 2017. Prevalence of Mycobacterium tuberculosis in Children Suffering from Lower Respiratory Tract Infection Attending a Tertiary Care Hospital, Hyderabad. Int.J.Curr.Microbiol.App.Sci. 6(7): 2567-2576.

doi: https://doi.org/10.20546/ijcmas.2017.607.303 